\title{
Copy-Number Variations in EYS: A Significant Event in the Appearance of arRP
}

\author{
Juan I. Pieras, ${ }^{1,2}$ Isabel Barragán, ${ }^{1,2}$ Salud Borrego, ${ }^{1,2}$ Isabelle Audo, $, 3,4,6$ \\ María González-Del Pozo, ${ }^{1,2}$ Sara Bernal, ${ }^{2,7}$ Montserrat Baiget, $^{2,7}$ Christina Zeitz, $^{3,4,5}$ \\ Shomi S. Bhattacharya, ${ }^{*, 8}$ and Guillermo Antiñolo*,1,2
}

Purpose. Autosomal recessive retinitis pigmentosa (arRP) has recently been associated with mutations in a novel gene, $E Y S$, which is a major gene for this disease. All published mutations so far are based on conventional PCR and are not adequate to identify midsized DNA rearrangements. This study was conducted to establish the prevalence of copy-number variations (CNVs) in the EYS gene in a cohort of arRP patients, including individuals in whom only one pathogenic change was detected by PCR-based sequencing.

Methods. A multiple ligation-dependent probe amplification (MLPA) was used for the molecular genetic analyses of CNVs by a novel EYS-specific kit. PCR-based direct sequencing was used in families where a pathogenic deletion or duplication was identified in one allele. Bioinformatics analyses was undertaken to study the effect of the mutations on protein structure and function.

RESUlTs. Six novel pathogenic CNVs were identified. Also, the presence of four midsized deletions was confirmed in patients

From the ${ }^{1}$ Unidad de Gestión Clínica de Genética, Reproducción y Medicina Fetal. Instituto de Biomedicina de Sevilla (IBIS), Hospital Universitario Virgen del Rocío/CSIC/Universidad de Sevilla, Seville, Spain; ${ }^{2}$ Centro de Investigación Biomédica en Red de Enfermedades Raras (CIBERER), Valencia, Spain; ${ }^{3}$ INSERM, U968, Paris, France; ${ }^{4}$ CNRS, UMR 7210. Paris, France; ${ }^{5}$ UPMC Univ. Paris 06, UMR_S 968 Institut de la Vision, Paris, France; ${ }^{6}$ Centre Hospitalier National d'Ophtalmologie des Quinze-Vingts, INSERM-DHOS, CIC 503, Paris, France; ${ }^{7}$ Servei de Genética, Hospital de la Santa Creu i Sant Pau, Barcelona, Spain; and ${ }^{8}$ Department of Cellular Therapy and Regenerative Medicine, Andalusian Molecular Biology and Regenerative Medicine Centre (CABIMER), Seville, Spain.

Supported by PN de I+D+I 2008-2011, Instituto de Salud Carlos III (ISCIII), Subdirección General de Evaluación y Fomento de la Investigación, Fondo de Investigación Sanitaria (PI081131), Spain; Consejería de Innovación, Ciencia y Empresa (PI08-CTS-03687), Junta de Andalucía, Spain; The Foundation Fighting Blindness (USA). The British Retinitis Pigmentosa Society. El Centro de Investigación Biomédica en Red de Enfermedades Raras is an initiative of the Instituto de Salud Carlos III. JIP was supported by Fondo de Investigación Sanitaria, and MG-DP by Consejería de Innovación, Ciencia y Empresa, Junta de Andalucía, Spain.

Submitted for publication January 27, 2011; revised March 7, 2011; accepted March 16, 2011.

Disclosure: J.I. Pieras, None; I. Barragán, None; S. Borrego, None; I. Audo, None; M. González-Del Pozo, None; S. Bernal None; M. Baiget, None; C. Zeitz, None; S.S. Bhattacharya, None; G. Antiñolo, None

"Each of the following is a corresponding author: Guillermo Antiñolo, Unidad de Gestión Clínica de Genética, Reproducción y Medicina Fetal, Hospital Universitario Virgen del Rocío, Avenida Manuel Siurot s/n, 41013, Seville, Spain; guillermo.antinolo.sspa@juntadeandalucia.es.

Shomi Bhattacharya, Department of Cellular Therapy and Regenerative Medicine, Andalusian Molecular Biology and Regenerative Medicine Centre (CABIMER), Avda. Americo Vespucio s/n. Edif. CABIMER, Parque Científico y Tecnológico Cartuja 931092, Seville, Spain;

shomi.bhattacharya@cabimer.es. previously identified. Midsized genomic rearrangements in EYS are disease causing in $\sim 4 \%$ of the families with no reported mutations and constitute the second pathogenic variation in $\sim 15 \%$ of cases where a mutation has been detected by direct sequencing

Conclusions. This is the first report of a systematic CNV screening of EYS gene in a cohort of arRP patients. Results suggest that midsized genomic rearrangements in EYS gene would be a common event in the appearance of RP phenotype. An efficient and cost-effective strategy validating a novel MLPA kit as a complementary diagnostic method for EYS pathogenic evaluation has been demonstrated. (Invest Ophthalmol Vis Sci. 2011;52:5625-5631) DOI:10.1167/iovs.11-7292

$\mathbf{R}$ etinitis pigmentosa (RP [MIM 268000]) is a generic name for inherited retinal dystrophies with loss of photoreceptor cells and retinal pigment deposits at midperiphery of the retina, which are visible on fundus examination. RP is characterized by primary degeneration of the rod photoreceptors followed by secondary degeneration of cones. This degeneration initially causes night blindness, followed by constriction of the visual field, abnormal color vision, and progressive loss of visual acuity in the later life. ${ }^{1}$ Prevalence of RP is approximately $1 / 4000$ births with over 1 million individuals affected worldwide. ${ }^{2}$ This disease can be inherited as an autosomal dominant, autosomal recessive, or an X-linked trait. All genes identified to date are believed to account for roughly $50 \%$ of all retinal dystrophy cases. ${ }^{3}$ The autosomal recessive form of RP is the commonest worldwide, accounting for approximately $50 \%$ $60 \%$ of cases. $^{2}$ To date, a total of 32 genes have so far been implicated in autosomal recessive RP (arRP; http://www.sph.uth.tm.edu/Retnet/). However, all together these genes appear to account for only $35 \%-45 \%$ of total arRP cases. ${ }^{2}$

Recently we have identified a new gene, EYS, corresponding to the RP25 locus, ${ }^{4}$ encoding an ortholog of Drosophila spacemaker (SPAM), as a commonly mutated gene in arRP. ${ }^{5}$ Spanning over $2 \mathrm{Mb}$ within the RP25 locus (6q12.1-6q15), EYS is the largest eye-specific gene identified so far. The longest $E Y S$ isoform encodes a protein of 3165 amino acids. Immunohistochemical data reveal its localization in the outer segment of the photoreceptor layer. Although its role in visual function has not yet been established, the evolutionary data, the new extensive bioinformatic analyses, and the known function of the drosophila ortholog support a structural role for this new protein contributing to the human retinal morphogenesis and architecture., 6 The initial identification of six independent mutations in our cohort of Spanish families linked to $R P 25$, together with the presence of different mutations in arRP families from different origins, supports EYS as the first major gene for arRP. ${ }^{5,7-12}$

The identification of EYS causative mutation is important for clinical diagnosis, predictions of the clinical course of the disease, genetic counseling, and future gene-targeted therapies. Majority 
of mutations identified so far include missense, nonsense, microdeletions and insertions, and putative pathogenic changes in the 5'UTR region. However, no duplications and few midsized deletions affecting EYS (to a few $\mathrm{Kb}$ ) have been reported to date. This low rate of identified copy-number variations (CNVs) leading to arRP may be explained by the fact that the techniques usually used, based on conventional PCR, are not adequate to identify such rearrangements. Due to the technical difficulties in determining gene dosage in this size range, it is possible that the proportion of mutations that are deletions or duplications has been underreported, and the presence of midsized rearrangements undetectable by PCR-based procedures would also explain the failure to detect the second mutant allele in a significant number of arRP families. ${ }^{7,9,10}$ In fact, only three studies have investigated midsized insertions/deletions in EYS using comparative genomic hybridization (CGH), CNVs analysis (Genechip 6.0 Affymetrix array; Affymetrix, Santa Clara, CA) and/or self-designed multiple ligation probe-dependent amplification (MLPA) probes. $5,7,9$

Thus, the availability of a rapid accurate molecular diagnostic complementary method to identify midsized rearrangements could enhance acute clinical management as well as genetic counseling. Here we report the pathogenic implication of EYS CNVs in Spanish and French arRP populations using MLPA. For this purpose we have tested and validated a novel EYS MLPA kit (MRC-Holland, Amsterdam, the Netherlands) in 95 arRP patients, including those where only one pathogenic change had previously been identified by PCR-based direct genomic sequencing. We have identified novel EYS rearrangements in six arRP cases. In addition, we have confirmed the presence of CNVs in four families with midsized deletions previously detected by CGH and self-designed MLPA.

\section{Materials AND Methods}

\section{Subjects and Clinical Data}

The study cohort comprises 95 Spanish and French unrelated patients affected by arRP, comprising 78 Spanish and 17 French patients. A full ophthalmic examination was performed, and the clinical diagnosis was based on visual acuity, fundus photography,

TABLE 1. arRP Families with One Likely Pathogenic Change Included in MLPA Studies in Order to Find a Second Mutant Allele

\begin{tabular}{|c|c|c|c|}
\hline $\begin{array}{l}\text { Family } \\
\text { ID }\end{array}$ & $\begin{array}{c}\text { DNA Change } \\
\text { Previously } \\
\text { Reported by } \\
\text { Direct } \\
\text { Sequencing }\end{array}$ & $\begin{array}{l}\text { Reference of } \\
\text { the Variation }\end{array}$ & $\begin{array}{c}\text { DNA Change } \\
\text { Identified by MLPA }\end{array}$ \\
\hline F51 & p.Trp558X & Audo et al. ${ }^{9}$ & - \\
\hline F228 & p.Cys1001X & Audo et al. ${ }^{9}$ & c. $(-340-?-748+? \mathrm{del})$ \\
\hline F444 & p.Trp2783X & Audo et al. ${ }^{9}$ & - \\
\hline F618 & p.Cys183AlafsX74 & Audo et al. ${ }^{9}$ & c. $\left(1300-? \_1459+? d e l\right)$ \\
\hline F311 & p.Pro2265GlnfsX46 & Audo et al. ${ }^{9}$ & - \\
\hline F360 & p.Ser1610PheFsX 46 & Audo et al. ${ }^{9}$ & c. $(6572-? 66725+? \mathrm{del})$ \\
\hline F109 & p.Asp1682Tyr & Audo et al. ${ }^{9}$ & - \\
\hline F393 & p.Leu2189Pro & Audo et al. ${ }^{9}$ & - \\
\hline F481 & p.Gly 2907 Glu & Audo et al. ${ }^{9}$ & - \\
\hline F116 & p.Cys1176Arg & Audo et al. ${ }^{9}$ & - \\
\hline F649 & p.Cys2139Tyr & Audo et al. ${ }^{9}$ & - \\
\hline F715 & p.Asp 1682Tyr & Audo et al. ${ }^{9}$ & - \\
\hline F123 & p.Asn 745 Ser & Audo et al. ${ }^{9}$ & - \\
\hline F85 & c. $2023+1 G>C$ & Audo et al. ${ }^{9}$ & - \\
\hline F221 & p.Pro1739Leu & Audo et al. ${ }^{9}$ & - \\
\hline RP60 & p.Gln27ArgfsX16 & Barragán et al. ${ }^{7}$ & - \\
\hline RP81 & p.Asn 745 Ser & Barragán et al. ${ }^{7}$ & - \\
\hline RP33 & p.Trp $1484 \mathrm{X}$ & Barragán et al. ${ }^{7}$ & - \\
\hline RP383 & p.Glu2503Lys & Barragán et al. ${ }^{7}$ & - \\
\hline VRP8 & p.Trp1484Arg & Barragán et al. ${ }^{7}$ & - \\
\hline
\end{tabular}

Table 2. List of Probes to Detect CNVs in the EYS Gene

\begin{tabular}{|c|c|c|c|}
\hline EYS Exon & $\begin{array}{c}\text { Ligation Site } \\
\text { NM_001142800.1 }\end{array}$ & $\begin{array}{c}\text { Distance to } \\
\text { Next Probe } \\
\text { (Kb) }\end{array}$ & \\
\hline Exon 1 & $6 \mathrm{nt}$ after exon 1 & 66.8 & \\
\hline Intron 1 & 415 nt before exon 2 & 144.1 & \\
\hline Exon 2 & - & & No probe \\
\hline Exon 3 & 173 nt before exon 3 & 0.8 & \\
\hline Exon 4 & $553-554$ & 1.2 & \\
\hline Intron 4 & $463 \mathrm{nt}$ after exon 4 & 92.1 & \\
\hline Exon 5 & - & & No probe \\
\hline Exon 6 & - & & No probe \\
\hline Intron 7 & $419 \mathrm{nt}$ after exon 7 & 17.6 & \\
\hline Exon 8 & $1806-1807$ & 30.9 & \\
\hline Exon 9 & $1935-1936$ & 18.4 & \\
\hline Exon 10 & - & & No probe \\
\hline Exon 11 & 2201-2202 & 39.2 & \\
\hline Exon 12 & $2509-2510$ & 0.2 & \\
\hline Exon 12 & 177 nt after exon 13 & 238.1 & \\
\hline Exon 13 & $2673-2674$ & 60.0 & \\
\hline Exon 14 & $2743-2742$ reverse & 51.8 & \\
\hline Exon 15 & $2889-2890$ & 33.2 & \\
\hline Exon 16 & $3007-3008$ & 9.9 & \\
\hline Exon 17 & 233 nt before exon 17 & 0.3 & \\
\hline Exon 17 & $3262-3263$ & 15.7 & \\
\hline Exon 18 & - & & No probe \\
\hline Exon 19 & $3503-3504$ & 64.0 & \\
\hline Exon 20 & $3632-3633$ & 1.0 & \\
\hline Exon 21 & $3743-3744$ & 8.1 & \\
\hline Exon 22 & $3810-3811$ & 187.6 & \\
\hline Exon 23 & 132 nt after exon 23 & 8.5 & \\
\hline Exon 24 & $4146-4147$ & 24.4 & \\
\hline Exon 25 & 4415-intron 25 & 2.0 & \\
\hline \multirow[t]{2}{*}{ Exon 26} & $5328-5329$ & 153.5 & \\
\hline & $1.6 \mathrm{~Kb}$ after exon 27 & 0.9 & \\
\hline Intron 27 & $1.3 \mathrm{~Kb}$ before exon 28 & & \\
\hline Intron 27 & 367 nt before exon 28 & 0.4 & \\
\hline Exon 28 & $6398-6399$ & 47.5 & \\
\hline Exon 29 & $6540-6541$ & 80.5 & \\
\hline Exon 30 & $6710-6731$ & 76.2 & \\
\hline Exon 31 & 6798-6799 & 164.5 & \\
\hline Exon 32 & - & & No probe \\
\hline Exon 33 & $28 \mathrm{nt}$ after exon 33 & 67.2 & \\
\hline Exon 34 & $\begin{array}{l}1 \mathrm{nt} \text { after exon } 34 \\
\text { reverse }\end{array}$ & 14.5 & \\
\hline Exon 35 & 7445-7444 reverse & 120.3 & \\
\hline Exon 36 & $7735-7736$ & 58.0 & \\
\hline Exon 37 & $8 \mathrm{nt}$ after exon 37 & 17.1 & \\
\hline Exon 38 & $12 \mathrm{nt}$ after exon 38 & 0.9 & \\
\hline Exon 39 & $8208-8209$ & 10.1 & \\
\hline Exon 40 & $8425-8426$ & 15.4 & \\
\hline Exon 41 & 8488-8489 & 35.8 & \\
\hline Exon 42 & 143 nt before exon 42 & 5.2 & \\
\hline Exon 43 & $8931-8932$ & & \\
\hline Reference probe & $18 \mathrm{q} 21.1$ & & \\
\hline Reference probe & 9q21.31 & & \\
\hline Reference probe & $4 \mathrm{p} 16.3$ & & \\
\hline Reference probe & $2 q 36.1$ & & \\
\hline Reference probe & $17 q 11.1$ & & \\
\hline Reference probe & 19p13.2 & & \\
\hline Reference probe & $22 q 12.3$ & & \\
\hline Reference probe & 6q23.3 & & \\
\hline
\end{tabular}

The eight reference probes were used as controls.

computerized testing of central and peripheral visual fields, and electroretinography (ERG) findings. RP was defined as bilateral visual loss, initial hemeralopy, restriction of visual field, gradual increased bone spicule pigmentation and decrease of visual acuity, attenuation of retinal vessels, reduced or undetectable electroretinogram (ERG), and waxy disc pallor. The pattern of inheritance in all participating patients was diagnosed based on the pedigree 
RP5

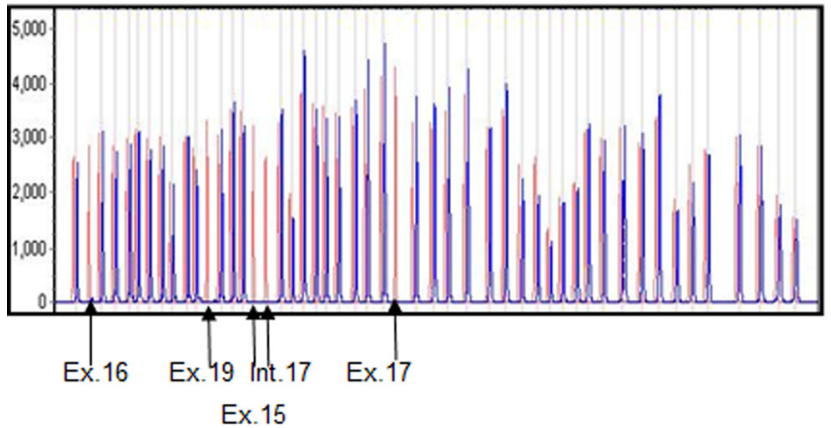

F115

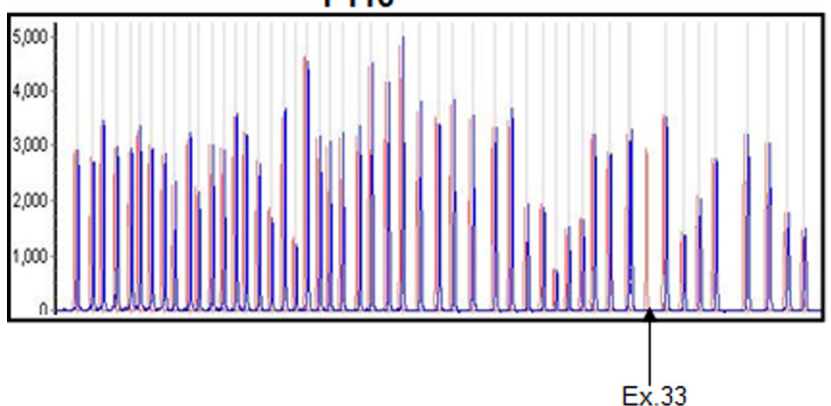

F735

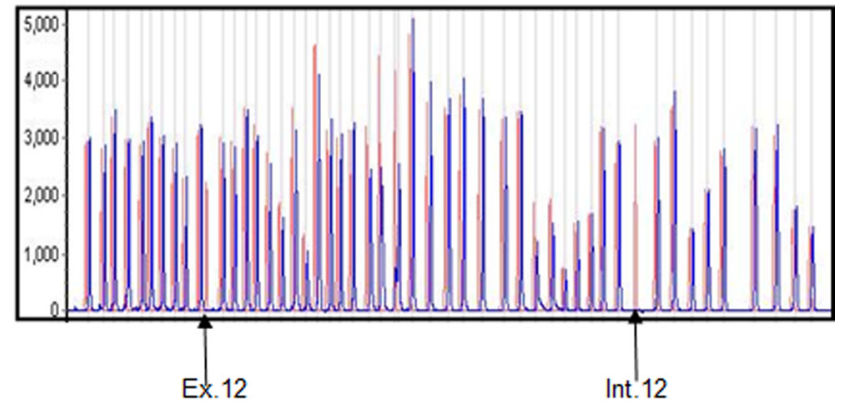

RP3

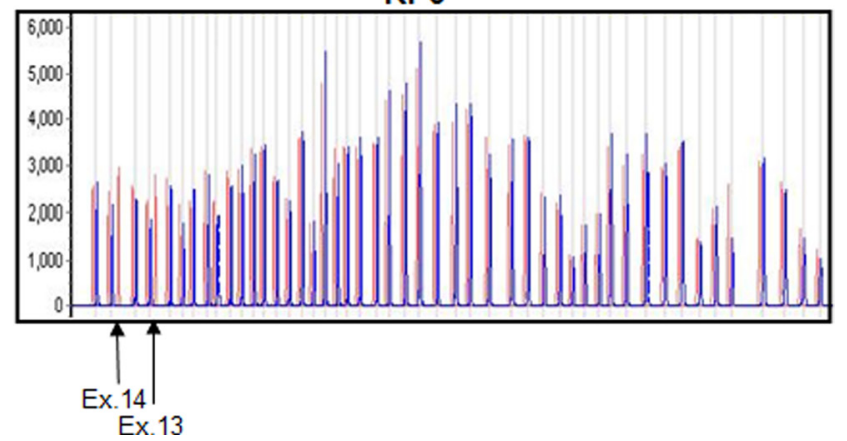

FIGURE 1. MLPA profiles for control individuals (red) and for arRP patients with homozygous deletions (blue). The absence of the peak of $E Y S$ was observed for exons 12, 13, 14, 15, 16, 17, 19, 33 and introns 12 and 17, indicated by arrows.

structure, characterized by more than one affected member and the nonaffected progenitors in arRP families.

Globally, our cohort included 71 arRP patients with no known mutation, 20 patients with a single mutation detected by PCR-based direct sequencing, and four positive controls for CNVs. Also three negative controls were included for technique normalization.

The Spanish cohort included five carriers of a pathogenic variant, previously identified in a heterozygote state by PCR-based direct sequencing. ${ }^{7}$ (Table 1). Additionally, two midsized EYS deletion carriers previously identified were included as positive controls. ${ }^{5}$ (Table 1 ).

The French cohort was composed of 15 patients with a previously identified heterozygous EYS mutation, ${ }^{9}$ (Table 1) and included two other homozygous individuals with midsized deletions as positive controls for the validation of MLPA.
In addition, available samples of probands' family members were tested for cosegregation studies. An informed consent was obtained from all participants for clinical and molecular genetic studies. The study conformed to the tenets of the Declaration of Helsinki.

\section{MLPA Analysis}

Gene dosage variation in EYS were analyzed by MLPA technology using a novel MLPA kit especially designed for EYS. The P328-X1 EYS MLPA Kit (MRC-Holland, Amsterdam, the Netherlands) contains 50 MLPA probes designed to detect alterations in the copy number of one or more exons in the EYS gene that can be implicated in arRP. In addition, this kit also includes eight reference control probes that hybridize to sequences in different regions of the genome (Table 2). The MLPA

TABLE 3. Copy-Number Variations and Mutations Identified by PCR-Based Direct Sequencing of EYS Gene in Spanish and French Families

\begin{tabular}{|c|c|c|c|c|c|}
\hline Family ID & Exon & DNA Change & Protein Change & Type of Change & Reference for the Variation \\
\hline \multirow[t]{2}{*}{ F228 } & $3-4$ & Deletion & c. $(-340-?-748+? \mathrm{del})^{*}$ & Heterozygous & This study \\
\hline & 20 & c. $3003 \mathrm{~T}>\mathrm{A}$ & p.Cys $1001 \mathrm{X}$ & Heterozygous & Audo et al. ${ }^{9}$ \\
\hline \multirow[t]{2}{*}{ RP1 } & $3-4$ & Duplication & c. $(-340-?, 748+? d u p)^{*}$ & Heterozygous & This study \\
\hline & 43 & c. $8897 \mathrm{G}>\mathrm{A}$ & pGly 2945 Glu & Heterozygous & This study \\
\hline \multirow[t]{2}{*}{ F618 } & 4 & c. $547 \mathrm{delT}$ & p.Cys183AlafsX74 & Heterozygous & Audo et al. ${ }^{9}$ \\
\hline & 9 & Deletion & c. $\left(1300-? \_1459+? d e l\right) \dagger$ & Heterozygous & This study \\
\hline \multirow[t]{2}{*}{ RP73 } & 12 & Deletion c.(1767-?_2023+?del) & p.Cys590TyrfsX4 & Heterozygous & Abd-El Aziz et al. ${ }^{5} /$ This study \\
\hline & 28 & c. $5857 \mathrm{G}>\mathrm{T}$ & p.Glu1953X & Heterozygous & Abd-El Aziz et al. ${ }^{5}$ \\
\hline F735 & 12 & Deletion c.(1767-?_2023+?del) & p.Cys590TyrfsX4 & Homozygous & Audo et al. ${ }^{9} /$ This study \\
\hline RP3 & $13-14$ & Deletion c.(2024-?_2259+?del) & p.Gly676GlufsX9del & Homozygous & This study \\
\hline RP5 & $15-19$ & Deletion c.(2260-?_2992+?del) & p.Ser754AlafsX6 & Homozygous & Abd-El Aziz et al. ${ }^{5} /$ This study \\
\hline RP2 & $16-19$ & Deletion c.(2382-?_2992+?del) & p.Cys795HisfsX 4 & Heterozygous & This study \\
\hline \multirow[t]{2}{*}{ F360 } & 26 & c. 4827 4830delTTCA & p.Ser1610PhefsX7 & Heterozygous & Audo et al. ${ }^{9}$ \\
\hline & 33 & Deletion & c. $\left(6572-? \_6725+? \mathrm{del}\right) \ddagger$ & Heterozygous & This study \\
\hline \multirow[t]{2}{*}{ F115 } & $32-33$ & Deletion & p.Asp2142AlafsX14 & Homozygous & Audo et al. ${ }^{9} /$ This study \\
\hline & & c.(6425-?_6725+?del) & & & \\
\hline
\end{tabular}

* There are no probes to detect possible deletions/duplications in exons 5 and 6 .

$\uparrow$ There are no probes to detect possible deletions in exon 10.

$\ddagger$ There are no probes to detect possible deletions in exon 32 . 
reaction was performed in a thermal cycler (Biometra TGRADIENT; Biometra, Goettingen, Germany), following the manufacturer's recommendations (http://www.mlpa.com).

Capillary electrophoresis analysis was performed in an analyzer (3730 DNA analyzer; Applied Biosystems, Foster City, CA), and commercially available software (GeneMarker v. 1.75; Softgenetics, State College, PA) was used for data analysis. We normalized the samples comparing the peak heights of patients versus controls. In addition, we included positive controls harboring three homozygous arRP EYS deletion patients (F115, F735, and RP5) and one patient with a heterozygous deletion (RP73), ${ }^{5,9}$ The MLPA analysis criteria were as follows: (1) normal if the individual dosage quotient values are within 0.8-1.0; (2) deletions or duplications if the dosage quotient values are around 0.5 or 1.5 , respectively; and (3) the mean SD of all samples for each peak should be below $10 \%$.

\section{PCR-Based Direct Genomic Sequencing of EYS}

To find the second pathogenic variation in those families where a new deletion/duplication was identified in one of the alleles by MLPA, we sequenced $E Y S$ by PCR-based direct sequencing. Moreover, these results helped to discard the presence of allelic variants in the probe target sequences that may have led to false positive results. PCR and sequencing procedures follows the protocols previously described in Barrágan et al. ${ }^{7}$.

To evaluate the pathogenicity of the novel variants, software (Netphos2.1; NetPhos2.0 Server, http://www.cbs.dtu.dk/services/NetPhos/) was used to predict the alteration in phosphorylation. ${ }^{13}$
All the changes were assigned a nucleotide number starting at the first translation base of EYS isoform according to the GenBank Reference Sequence Version FJ416331; GI: 212675237; Transcript Reference Sequence: NM_001142800.1 (ensemble entry ENST00000503581).

\section{RESUlts}

\section{Validation of EYS P328-X1 MLPA Kit}

Using a novel MLPA P328-X1 Kit, we first confirmed the presence of four midsized deletions in patients previously identified by CGH and self-designed MLPA. ${ }^{5,9}$ They consisted of three midsized homozygous deletions and one patient with a heterozygous deletion affecting exons 15-19, 32-33, and 12, in control families RP5, F115, F735, and RP73, respectively (Fig. 1, Table 3).

\section{Identification of Novel Very Likely Pathogenic CNVs}

MLPA analyses led to the identification of six novel EYS rearrangements. The sequence variants were designated in accordance with the Human Genome Variation Society recommendations (http://www.hgvs.org/mutnomen/) (Table 1).

In the RP1 family, we found a heterozygous duplication of exons 3 and 4 in the affected individual (Fig. 2). It is the first
RP73

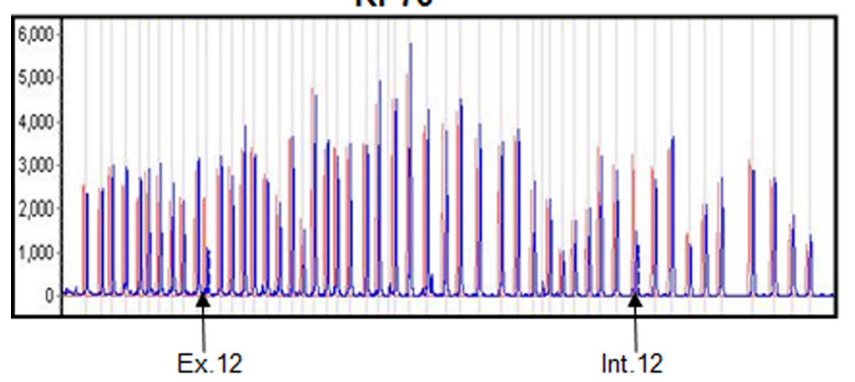

RP2
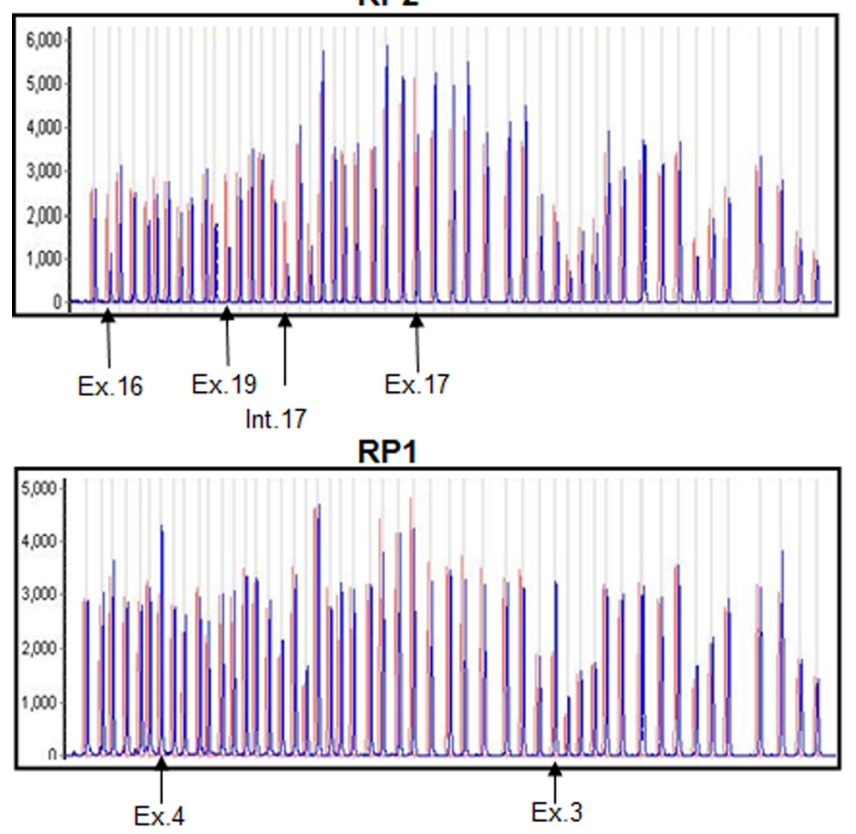

F228

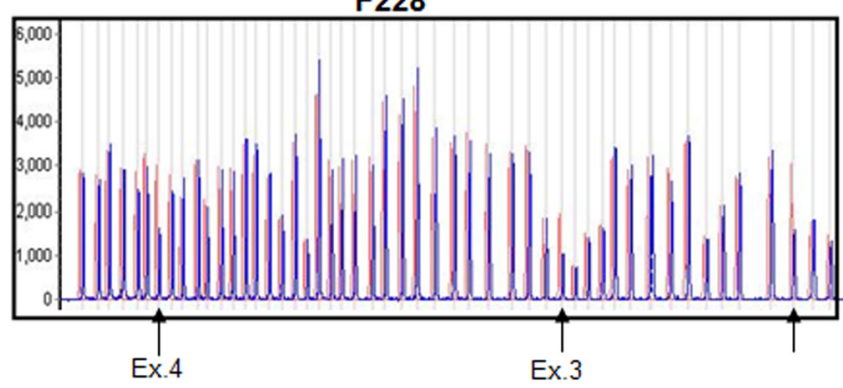

F618

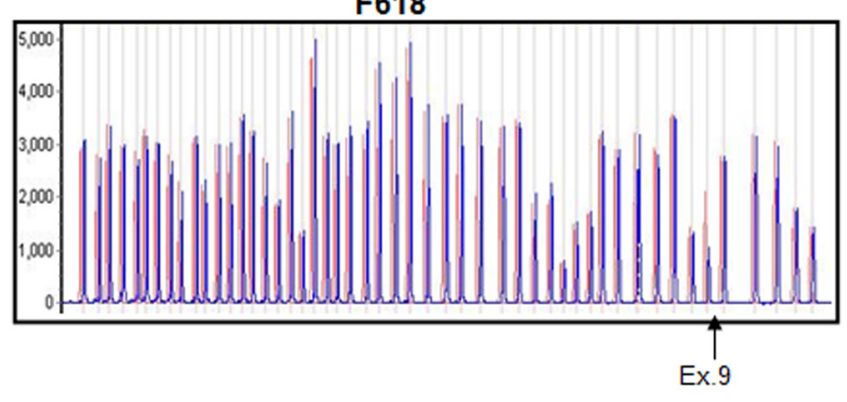

F360

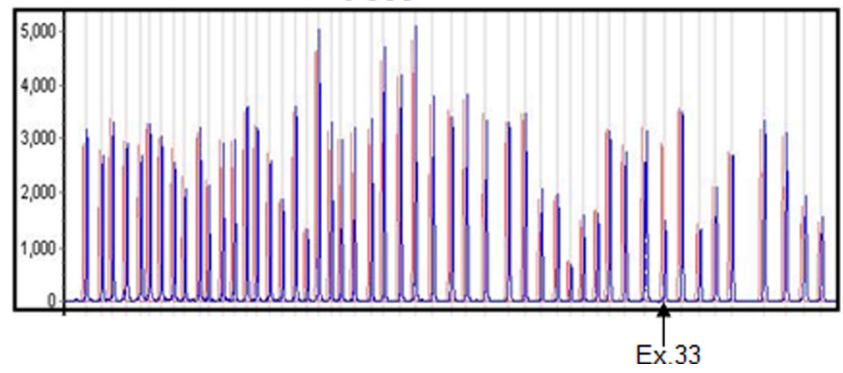

FigURE 2. MLPA profiles for control individuals (red) and for arRP patients with heterozygous deletions (blue). For the RP patients a decrease of dosage was observed for EYS exons 3, 4,12,16,17, 19, 33 and introns 4, 12, and 17, indicating a heterozygous deletion. In the case of RP1 family, an increment for the dosage of EYS exons 3 and 4 indicates a heterozygous duplication for these exons. 
A

\section{p.Gly2945Glu}

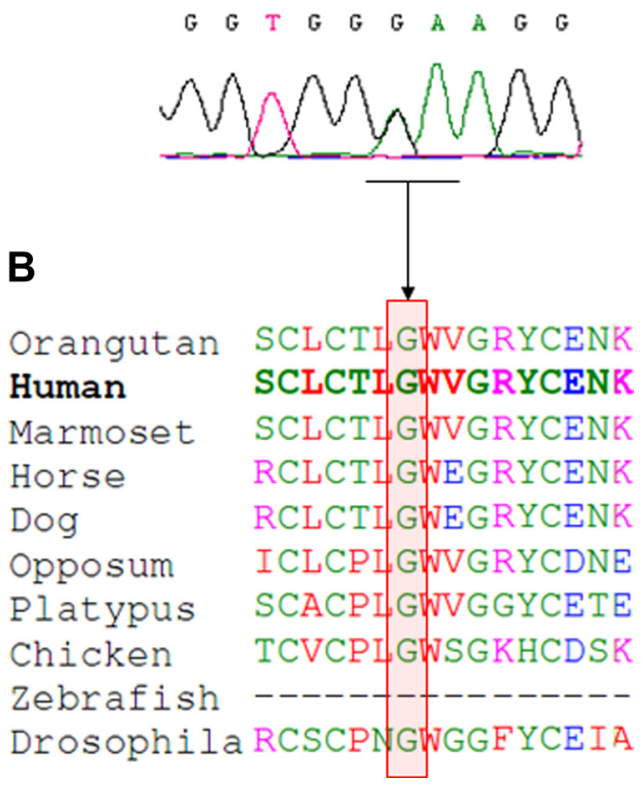

FIGURE 3. (A) Electropherogram of EYS mutation at nucleotide position c.8897G $>$ A in the proband of family RP1. (B) Alignment of the SPAM peptide sequence in human and in other species using CLUSTAL W (1.82) multiple sequence alignment.

time a duplication in EYS is reported.

Direct sequencing of this patient's DNA revealed a c.8897G $>$ A (p.Gly2945Glu) as the second pathogenic change (Fig. 3). The amino acid substitution locates in an EGF domain of SPAM. Turning to evolutionary conservation, this glycine is present in this position in EYS homologues previously reported by our group. ${ }^{7}$ The substitution of glycine to glutamate in position 2945 introduces an acidic polarity in a previously hydrophobic position. In addition, this change is not tolerated according to computational predictions, since it implies the loss of one phosphorylation site (NetPhos2.1).

In the RP3 family index patient, we found a novel homozygous deletion including exons 12 and 13. The segregation analysis in the family was consistent with the recessive pattern of inheritance (Table 4).

Index patients in $F 225, F 618$, and $F 360$ families had been previously described as carrying truncating mutations identified by PCR-based direct sequencing (Table 1). After MLPA screening, we have identified three midsized heterozygous deletions, affecting exons 3-4, 9, and 33, respectively, as the second pathogenic change in the $E Y S$ gene. In addition, family segregation in available members (F618 and F360), supported the pathogenic nature of those mutations (Table 4).

The screening of the index patient from family $R P 2$ revealed a heterozygous deletion involving exons 16-19. However, in this case, PCR-based direct sequencing failed to identify the second mutant allele.

\section{Discussion}

Molecular diagnosis of RP is a challenging task given the important genetic heterogeneity of these groups of diseases. EYS represents a major arRP gene, as can be appreciated in view of the high number of EYS mutations detected by PCR-based direct genomic sequencing reported in different arRP patients and the diverse ethnic origins of these families. ${ }^{5,7-12}$ However, mutations due to midsized deletions (a few kilobases) in EYS have seldom been reported.

Typical screening protocols using conventional PCR-based sequencing methods are only able to detect small duplications/ deletions (a few base pairs) and are dependent on the size of the amplified fragment in one reaction ( $\sim 1 \mathrm{~Kb}$ maximum) On the other hand, cytogenetic techniques can exclusively detect large alterations ranging several megabases in size. None of the

TABLE 4. Segregation of Pathogenic EYS Mutations in arRP Patients and Family Members

\begin{tabular}{|c|c|c|c|c|c|c|}
\hline $\begin{array}{l}\text { Index Patient, } \\
\text { Family Members }\end{array}$ & Family & Exon & Nucleotide Exchange & Protein effect & Allele State & Reference \\
\hline \multirow[t]{2}{*}{ CIC01001 } & \multirow[t]{2}{*}{ F618 } & 4 & c. $547 \mathrm{delT}$ & p.Cys183AlafsX74 & Het & Audo et al. ${ }^{9}$ \\
\hline & & 9 & Deletion & c. $\left(1300-? \_1459+? d e l\right)^{* *}$ & Het & This study \\
\hline Unaff. spouse & & 4 & - & - & - & Audo et al. ${ }^{9}$ \\
\hline CIC03487 & & 9 & - & - & - & This study \\
\hline Aff. sister & & 4 & c. $547 \mathrm{delT}$ & p.Cys183AlafsX74 & Het & Audo et al. ${ }^{9}$ \\
\hline CIC03499 & & 9 & Deletion & c. $\left(1300-? \_1459+? d e l\right)^{* *}$ & Het & This study \\
\hline Unaff. mother & & 4 & c. $547 \mathrm{delT}$ & p.Cys183AlafsX74 & Het & Audo et al. ${ }^{9}$ \\
\hline CIC03605 & & 9 & - & - & - & This study \\
\hline $03 / 2522 b$ & RP3 & $13-14$ & Deletion & p.Gly676GlufsX9del & Hom & This study \\
\hline Unaff. daughter & & $13-14$ & Deletion & p.Gly676GlufsX9del & Het & This study \\
\hline \multicolumn{7}{|l|}{$03 / 2523 b$} \\
\hline Unaff. son & & $13-14$ & Deletion & p.Gly676GlufsX9del & Het & This study \\
\hline \multicolumn{7}{|l|}{$03 / 2524 b$} \\
\hline Unaff. niece 12227 & & $13-14$ & Deletion & p.Gly676GlufsX9del & Het & This study \\
\hline Unaff. nephew & & $13-14$ & Deletion & p.Gly676GlufsX9del & Het & This study \\
\hline \multicolumn{7}{|l|}{12226} \\
\hline & F360 & 26 & c.4827_4830delTTCA & p.Ser1610PhefsX7 & Het & Audo et al. ${ }^{9}$ \\
\hline CIC00529 & & 33 & Deletion & c. $(6572-? ; 6725+? d e l)^{* * * *}$ & Het & This study \\
\hline Unaff. father & & 26 & c.4827_4830delTTCA & p.Ser1610PhefsX7 & Het & Audo et al. ${ }^{9}$ \\
\hline CIC00794 & & 33 & - & - & - & This study \\
\hline Unaff. mother & & 26 & - & - & - & Audo et al. ${ }^{9}$ \\
\hline CIC00528 & & 33 & Deletion & c. $\left(6572-?[6725+? d e l)^{\text {**** }}\right.$ & Het & This study \\
\hline Unaff. sister & & 26 & - & - & - & Audo et al. ${ }^{9}$ \\
\hline CIC00710 & & 33 & Deletion & c. $\left(6572-?[6725+? \mathrm{del})^{\text {**** }}\right.$ & Het & This study \\
\hline Unaff. sister & & 26 & c.4827_4830delTTCA & p.Ser1610PhefsX7 & Het & Audo et al. ${ }^{9}$ \\
\hline CIC00766 & & 33 & - & - & - & This study \\
\hline
\end{tabular}


two approaches are powerful enough and adequate to detect CNVs affecting specific regions corresponding to a few kilobases. There are some traditional techniques used to detect midsized deletions/duplications such as southern blot or quantitative/semiquantitative PCR, but they are expensive, time consuming, and not suitable for high-throughput results. For example, only a few samples can be run per gel by southern blot, and tests may take several days; using real-time PCR, the number or targets that can be interrogated in a reaction is limited by the number of fluorophores available. Also, the cost of buying fluorescently labeled probes for every intended target can be prohibitive.

For these reasons we sought to perform CNV screening using MLPA technology (see Supplementary Material and Supplementary Fig. S1, http://www.iovs.org/lookup/suppl/doi:10.1167/ iovs.11-7292/-/DCSupplemental) in arRP patients, including the ones where only one pathogenic change had been identified by PCR-based direct sequencing.

The major advantages of MLPA are ease of use, high throughput, and being cheaper than other techniques such as real-time PCR or CGH. The MLPA method allows the detection of midsized insertions/deletions ranging from the probe size ( $\sim 40$ bases) to a few $\mathrm{Kb}$ (depending on the length of tested gene) in a large number of individuals within a short time period. This technique is being used extensively in the molecular diagnosis of a wide range of diseases caused by deletions or duplications of one or more exons in specific genes. ${ }^{14,15}$ In this case the novel MLPA kit has a maximum detection range of $\sim 2 \mathrm{Mb}$, corresponding to the EYS genomic interval.

First, to evaluate the novel MLPA kit, we confirmed the presence of four midsized deletions in patients previously identified by CGH and self-designed MLPA. ${ }^{5,9}$ Therefore, validation of the novel kit used was successfully achieved.

As a result of the MLPA screening for CNVs, we have identified six novel EYS rearrangements in six out of 91 unrelated patients with arRP (see Supplementary Fig. S2, http://www.iovs.org/lookup/ suppl/doi:10.1167/iovs.11-7292/-/DCSupplemental). Majority of identified CNVs are predicted to generate a frameshift leading to a premature stop codon, and probably, most of these altered mRNA transcripts will be degraded through nonsense mediated decay. ${ }^{16}$ In the case of F228, RP1, and F360 families, we cannot predict the putative protein effect of the mutation, since the extent of the CNVs is unknown, due to the absence of probes for the adjacent exons (see Supplementary Fig. S3, http://www.iovs.org/ lookup/suppl/doi:10.1167/iovs.11-7292/-/DCSupplemental). It is very likely that they would lead to a frame shift generating a novel stop codon, or the loss/gain of a significant portion of the protein, rich in important functional domains. In both cases the outcome would be sufficient to be pathogenic.

The identification of different CNVs in EYS in three out of 71 families with no known mutations, reveals a probable mutation frequency of $\sim 4 \%$ of the cases, indicating that this kind of genomic rearrangements in EYS gene would be a significant possibility in the appearance of the RP phenotype. Also, three out of 20 families carrying a mutation in one allele detected by PCR-based direct sequencing were found to have a rearrangement in the second mutated allele in EYS. This indicates that, at least, $15 \%$ of families with one heterozygous change identified by PCR-based direct sequencing would be a carrier of a midsized pathogenic deletion/insertion. However, it is still possible that some of the 17 remaining cases with only one pathogenic mutation also carry deletions/duplications affecting the EYS gene, as the current P328-X1 probemix does not contain probes for exons $2,5,6,10,18$, and 32 . Therefore, the $\mathrm{P} 328-\mathrm{X} 1$ probemix must be updated to improve both the accuracy and detection rate of rearrangements in EYS by MLPA.

In summary, this is the first report of a systematic CNV screening of EYS gene in a large cohort of arRP patients. Our results show that midsized genomic rearrangements in $E Y S$ are a common event in the development of the RP phenotype. They are responsible for the disease in about 4\% (3/71) of the families with no reported mutations and constitute the second pathogenic variation in approximately 15\% (3/20) of cases where a mutation has been detected by PCR-based direct sequencing. Globally, 5\% (5/91) of all arRP families included in this study bear a pair of EYS mutations as the likely cause of the disease, and $\sim 1 \%(1 / 91)$ present a heterozygous mutation. Of the mutations, $\sim 7 \%(6 / 91)$ are midsized rearrangements, whereas $\sim 2 \%(2 / 91)$ are single-nucleotide substitutions, and $\sim 2 \%(2 / 91)$ are other variants detectable by PCR-based sequencing (Fig. 4).

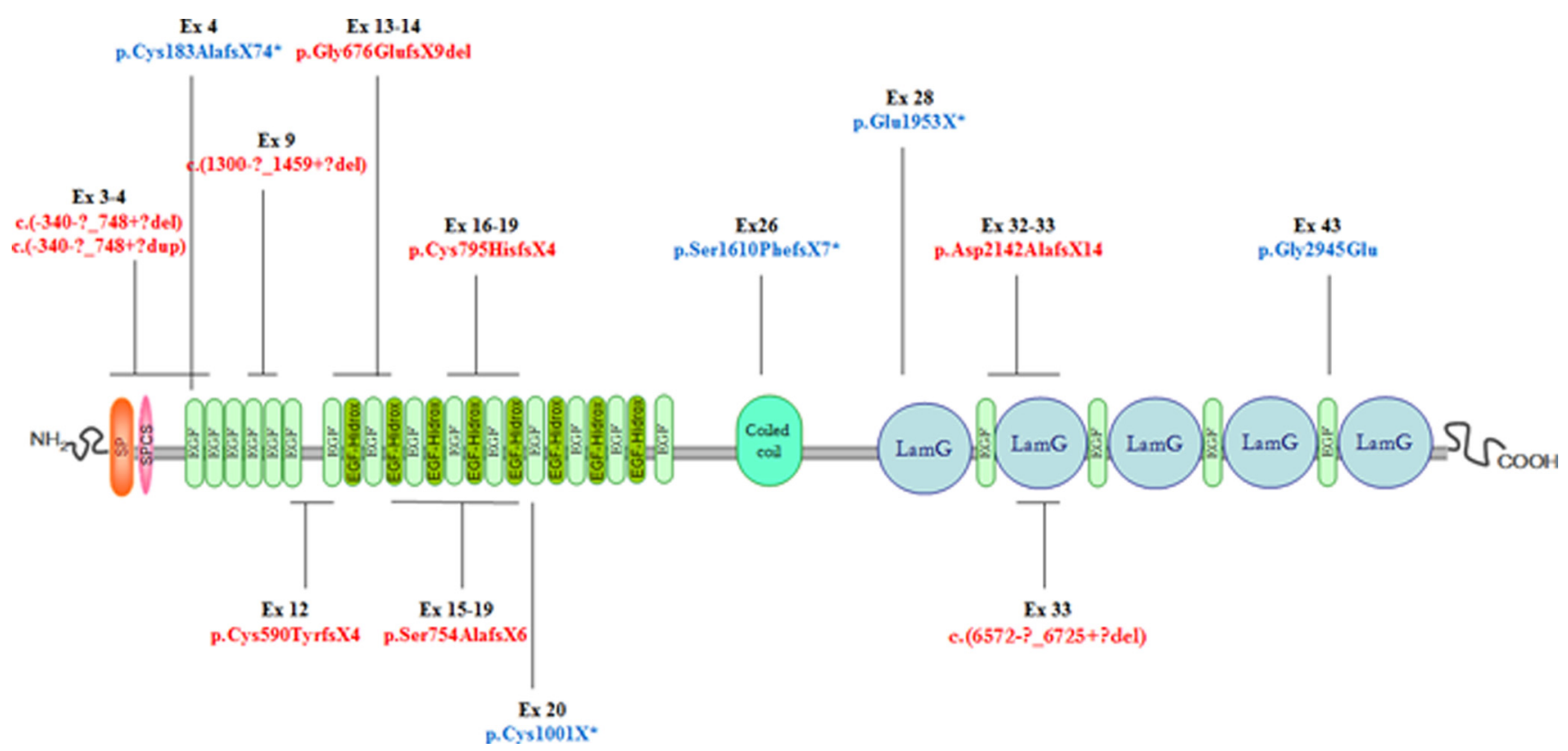

FIGURE 4. Distribution of EYS mutations along the domain structure of SPAM. Large rearrangements are indicated in red. Blue is used for depicting small deletions and single nucleotide changes. Mutations previously identified by PCR-based direct sequencing are indicated by an asterisk. 
Determination of the underlying genetic defects in a patient is a prerequisite for gene or mutation-specific therapy; thus, this knowledge is crucial for future therapies design. Therefore, the development of efficient and cost-effective strategies to detect mutation is mandatory. We have demonstrated that MLPA is an efficient and cost-effective strategy as a complementary diagnostic method for EYS pathogenic evaluation in arRP families.

\section{References}

1. Hamel C. Retinitis pigmentosa. Orphanet J Rare Dis. 2006;1:40.

2. Hartong DT, Berson EL, Dryja TP. Retinitis pigmentosa. Lancet. 2006;368:1795-1809.

3. Pomares E, Marfany G, Brión MJ, Carracedo A, Gonzalez-Duarte R. Novel high-throughput SNP genotyping cosegregation analysis forgenetic diagnosis of autosomal recessive retinitis pigmentosa and Leber congenital amaurosis. Hum Mutat. 2007;28:511-516.

4. Ruiz A, Borrego S, Marcos I, Antiñolo G. A major locus for autosomal recessive retinitis pigmentosa on $6 \mathrm{q}$, determined by homozygosity mapping of chromosomal regions that contain gammaaminobutyric acid-receptor clusters. Am J Hum Genet. 1998;62: 1452-1459.

5. Abd El-Aziz MM, Barragán I, O'Driscoll et al. EYS, encoding an ortholog of Drosophila spacemaker, is mutated in autosomal recessive retinitis pigmentosa. Nat Genet. 2008;40:1285-1287.

6. Zelhof AC, Hardy RW, Becker A, Zuker CS. Transforming the architecture of compound eyes. Nature. 2006: 12;443:696-699.

7. Barragán I, Borrego S, Pieras JI, et al. Mutation spectrum of EYS in Spanish patients with autosomal recessive retinitis pigmentosa. Hum Genet. 2010;31:E1772-1800.
8. Collin RW, Littink KW, Klevering BJ, et al. Identification of a $2 \mathrm{Mb}$ human ortholog of Drosophila eyes shut/spacemaker that is mutated in patients with retinitis pigmentosa. Am J Hum Genet. 2008;83:594-603.

9. Audo I, Sahel JA, Mohand-Saïd S, et al. EYS is a major gene for rod-cone dystrophies in France. Hum Mutat. 2010;31:E14061435.

10. Abd El-Aziz MM, O'Driscoll CA, Kaye RS, et al. Identification of novel mutations in the ortholog of Drosophila eyes shut gene (EYS) causing autosomal recessive retinitis pigmentosa. Invest Ophthalmol Vis Sci. 2010;51:4266-4272.

11. Bandah-Rozenfeld D, Littink KW, Ben-Yosef T, et al. Novel null mutations in the EYS gene are a frequent cause of autosomal recessive retinitis pigmentosa in the Israeli population. Invest Ophthalmol Vis Sci. 2010;51:4387-4394.

12. Littink KW, van den Born LI, Koenekoop RK, et al. Mutations in the EYS gene account for approximately $5 \%$ of autosomal recessive retinitis pigmentosa and cause a fairly homogeneous phenotype. Ophthalmology. 2010: 117:2026-2033, 2033, e1-7.

13. Blom N, Gammeltoft S, Brunak S. Sequence- and structure-based prediction of eukaryotic protein phosphorylation sites. J Mol Biol. 1999;294:1351-1362.

14. Aller E, Jaijo T, García-García G, et al. Identification of large rearrangements of the PCDH15 gene by combined MLPA and oligonucleotide CGH-array: large duplications are responsible for Usher syndrome. Invest Ophthalmol Vis Sci. 2010;51:5480-5485.

15. Sellner LN, Taylor GR. MLPA and MAPH: new techniques for detection of gene deletions. Hum Mutat. 2004;23:413-419.

16. Frischmeyer PA, van Hoof A, O'Donnell K, Guerrerio AL, Parker R, Dietz HC. An mRNA surveillance mechanism that eliminates transcripts lacking termination codons. Science. 2002;22:2258-2261. 